\title{
Magnetic resonance imaging findings before and after treatment of spontaneous intracranial hypotension
}

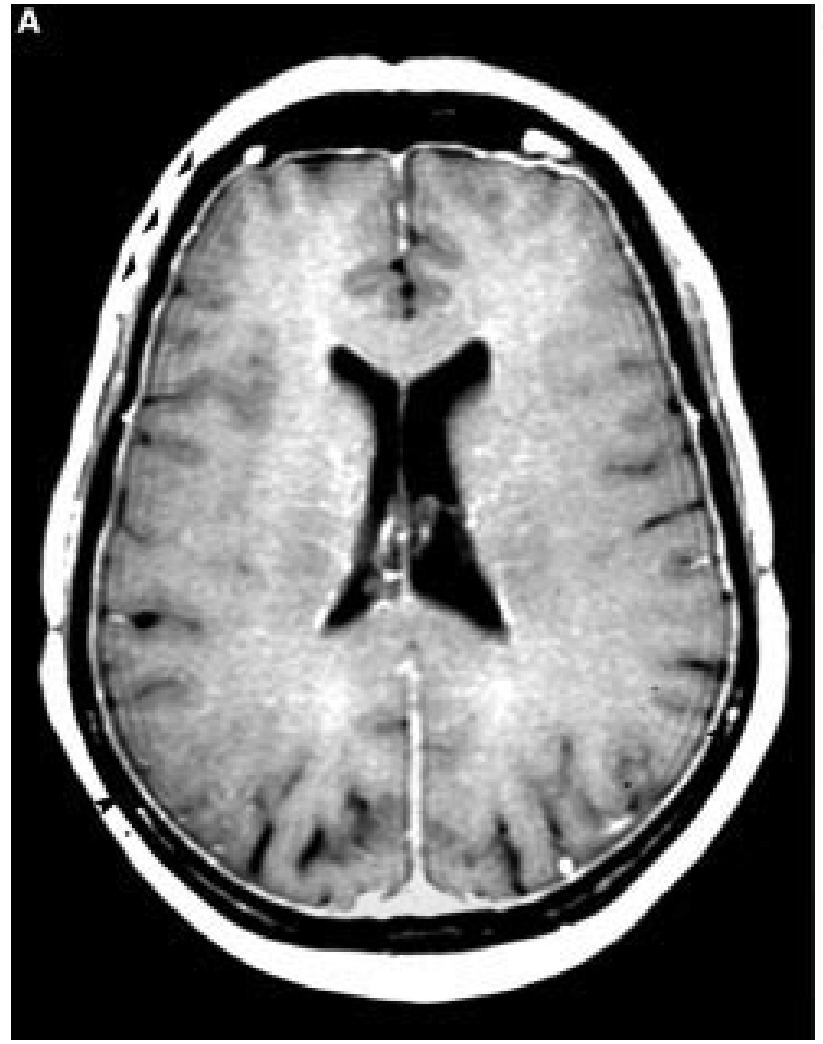

Figure 1 Axial sections of a T1 weighted MRI of the brain after gadolinium enhancement seen before treatment; (B) this had disappeared after treatment.

The syndrome of spontaneous intracranial hypotension is characterised by postural headache, low CSF pressure, and MRI findings of diffuse pachymeningeal gadolinium enhancement without a history of head or back trauma or lumbar puncture. ${ }^{1}$ We report on the MRI findings of a patient with spontaneous intracranial hypotension, before and after treatment.

A 44 year old woman presented with severe subacute headache, vomiting, and diplopia. Previously she had experienced a period of severe headache that lasted for some weeks and resolved spontaneously. Neurological examination showed a left sided abducens nerve palsy and was otherwise normal. A T1 weighted spin echo MRI image of the brain after gadolinium administration showed diffuse and homogeneous meningeal thickening and enhancement (fig $1 \mathrm{~A}$ ). To rule out meningitis a lumbar puncture was performed. The CSF opening pressure was 0 $\mathrm{cm} \mathrm{H}_{2} \mathrm{O}$ and fluid was obtained after a Vasalva manoeuvre. Analysis of CSF was normal, even after repeating the lumbar puncture. On inquiry the patient said that the headache was relieved when she spent a few minutes in a supine position and worsened when she was in an upright position. She had no history of lumbar puncture or head or back trauma. The diagnosis of spontaneous intracranial hypotension was established. Treatment consisted of an

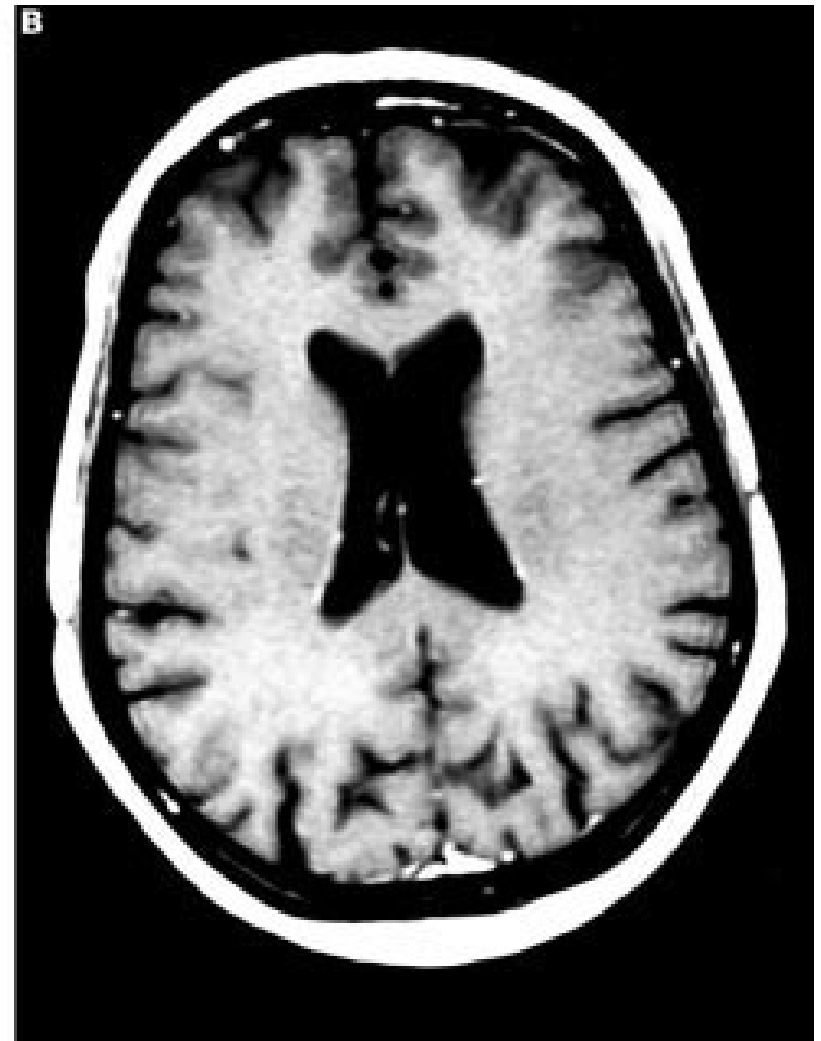

administration before and after treatment. (A) Extensive meningeal

extradural blood patch ( $20 \mathrm{ml}$ autologous blood) at a lumbar level and her headache and sixth nerve palsy resolved within 24 hours. A follow up MRI (fig 1 B) 3 weeks after the treatment showed no meningeal thickening and enhancement. The patient was seen 6 months after treatment and had had no recurrence.

Diffuse meningeal gadolinium enhancement on MRI in intracranial hypotension was first described by Morki. ${ }^{2}$ It is thought to be caused by compensatory venous vasodilation and therefore results in a greater concentration of gadolinium in the dural vasculature and interstial fluid. ${ }^{3}$ The MRI findings in this case demonstrate that diffuse meningeal enhancement can disappear after increasing CSF pressure by an extradural bloodpatch.

G J LUIJCKX

Department of Neurology, Maasland Hospital, PO Box 5500, 6130 MB Sittard, The Netherlands

T DE JAEGERE

Department of Radiology

1 Chung SJ, Kim JS, Lee MC. Syndrome of cerebral spinal fluid hypovolemia. Clinical and imaging features and outcome. Neurology 2000;55:1321-7.

Mokri B, Krueger BR, Miller GM, et al. Meningeal gadolinium enhancement in low pressure headaches. F Neuroimaging 1993;3:11.

3 Mokri B, Prisi JE, Scheithauer BW, et al. Menigeal biopsy in intracranial hypotension. Neurology 1995;45:1801-7. 